\title{
Cognitive Behaviour Therapy for psychosis: the impact of therapist training and supervision
}

Article

Accepted Version

Steel, C., Tarrier, N., Stahl, D. and Wykes, T. (2012) Cognitive Behaviour Therapy for psychosis: the impact of therapist training and supervision. Psychotherapy and Psychosomatics, 81 (3). pp. 194-195. ISSN 1423-0348 doi: https://doi.org/10.1159/000334250 Available at https://centaur.reading.ac.uk/24693/

It is advisable to refer to the publisher's version if you intend to cite from the work. See Guidance on citing.

To link to this article DOI: http://dx.doi.org/10.1159/000334250

Publisher: Karger

All outputs in CentAUR are protected by Intellectual Property Rights law, including copyright law. Copyright and IPR is retained by the creators or other copyright holders. Terms and conditions for use of this material are defined in the End User Agreement.

www.reading.ac.uk/centaur 
Central Archive at the University of Reading

Reading's research outputs online 
Running title: CBT for schizophrenia and therapist effects

Cognitive Behaviour Therapy (CBTp) for psychosis: The Impact of Therapist Training and Supervision

\author{
Craig Steel ${ }^{1}$, Nicholas Tarrier ${ }^{2}$, Daniel Stahl ${ }^{3}$ and Til Wykes ${ }^{2}$ \\ ${ }^{1}$ Department of Psychology and Clinical Language Sciences \\ University of Reading \\ ${ }^{2}$ Department of Psychology \\ Institute of Psychiatry, \\ King's College London \\ ${ }^{3}$ Department of Biostatistics \\ Institute of Psychiatry, \\ King's College London
}

Word Count: 981

Address for correspondence:

Dr Craig Steel, Department of Psychology and Clinical Language Sciences, University of Reading

Reading RG6 6AL

Tel: 0044 (0)118 378 7550; Fax: 0044 (0)118 378 6715; e-mail c.steel@ reading.ac.uk. 
The varied outcomes of clinical trials of cognitive behaviour therapy for psychosis (CBTp) have recently been reported, with the methodological rigour of the individual studies being associated with outcome [1]. However, issues relating to trial therapists training, experience and supervision may also be predictive of outcome. The aim of the current study is to report on a number of therapist effects within clinical trials of CBTp, and their relationship with outcome. Such dissection of the evidence base for CBTp is clearly important in helping to develop future training guidelines for evidence based interventions. The current report builds upon our recently published meta-analysis of CBTp [1], which included 34 trials of CBTp and reported a moderate effect size (approximately 0.4 ) for a variety of outcome measures, e.g. positive symptoms, negative symptoms and functioning. In order to reduce potential heterogeneity, we limited our current sample to include only those studies based on individual CBTp targeting positive symptoms, which form the vast majority of our current evidence base. We include a measure of trial methodology, the Clinical Trials Assessment Measure (CTAM) [2], in order to enable this variable to be included within analyses.

Given the lack of previous research upon which to base current hypotheses, we generated a list of 8 variables associated with training and supervision. 18 international experts, drawn from the Beck CBT for psychosis group, rated all 8 variables as important factors within clinical trials of CBTp. We re-ran the search criteria employed by Wykes et al. (2008), using the same entry criteria, to ensure our sample was up to date. Data for the main study were gathered by contacting the lead authors of the selected trials in order to obtain the relevant information for each trial. One study [3] was dropped from the current analyses, as data on training and supervision of trial therapists were not available, and one new study was included [4], leaving a final sample of 24 studies. 
The extraction of data relating to positive symptoms and the calculation of individual trial effect size was conducted as in our previous study [1]. We conducted an initial analyses which consisted of correlations between the 8 therapist and supervision variables, along with the participant characteristics of age, gender and number of hospitalisations, and effect size. We then assessed the predictive power of these variables on effect size in separate linear meta-regressions. Given that methodology has been shown to be a potent variable in relation to outcome [1] we investigated these within trial effects whilst controlling for methodological rigour.

Almost $70 \%$ of the combined caseload from all 24 trials was conducted by a therapist without a specific postgraduate qualification in CBT. About two-thirds of the overall caseload was conducted by clinical psychologists, with 13 of the 24 trials employing this profession alone. On average trial therapists received more than 4 hours of supervision per month, predominantly on an individual basis. Thirty-seven percent of the overall participants were seen by a therapist who was focussing their clinical work exclusively on a clinical trial.

There was a significant positive effect of the proportion of trial therapists clinical work which was exclusively focussed on trial cases, and an almost significant positive effect of the quantity supervision therapists received within a trial, on effect size after controlling for CTAM scores (see Table 1). The correlation coefficients of both these variables were above 0.4 and significant. They were not significantly correlated with each other $(r=0.22$; $\mathrm{p}=0.3$ ). All other correlations were 0.31 or smaller and non-significant.

INSERT TABLE 1 ABOUT HERE 
Our results suggest that therapists whose clinical work was predominantly focussed on a clinical trial achieved better outcomes. Clearly, the amount of clinical work dedicated to a clinical trial will be associated with other variables. However, a major strength of the current analyses was that it enabled overall trial methodology to be controlled for, suggesting that this is not the mediating variable. It is therefore seems that being able to focus on trial clinical work is associated with something that is yet to be measured, such as the development of specific skills which are not gained through generic clinical experience. Although there is much to learn within this area, it would seem important for clinicians to note that the best clinical outcomes are associated with those who dedicate most of their clinical work to a specific intervention. This may especially be the case within such a complex intervention as CBT for psychosis. Therefore, whilst it is important that clinicians receive appropriate training they also appear to need a reasonably high caseload in order to maintain best practice.

There was some support for more frequent supervision of trial therapists being associated with improved outcome, although a metaregression of the same relationship showed a result slightly outside of significance. While more frequent supervision may have occurred within the larger trials with more funds, the quality of trial methodology was controlled within the regression analyses, suggesting supervision frequency is likely to be an independent predictor of outcome. Further, the frequency of supervision was not related to the percentage of therapists' clinical work dedicated to a clinical trial, and these variables are therefore likely to have an independent effect on outcome. Our results highlight the need for further research in order to 'unpack' which therapist training and supervision characteristics are of significance in order to efficiently disseminate the protocols produced within clinical trials. In the meantime we suggest that when clinical trials of psychological interventions are 
published that the supervision arrangements should be detailed along with whether therapists were trial dedicated or not.

These results need to be treated in the context of multiple tests having been performed. Also, the limited variance within some of the variables included within the current study, and a moderate sample size of 24 only provides power to detect large effects. However, if effects are subtle they may not be important. However, there is need for caution when interpreting non-significant results.

\section{References}

1 Wykes T, Steel C, Everitt B, Tarrier N: Cognitive Behaviour Therapy for Schizophrenia: Effect Sizes, Clinical Models and Methodological Rigor. Schiz Bull 2008; 34: 523-537.

2 Tarrier N, Wykes T: Is there evidence that cognitive behaviour therapy is an effective treatment for schizophrenia? A cautious or cautionary tale? Beh Res Ther 2004; 42: $1377-1401$.

3 Milton F, Patwa VK, Hafner RJ: Confrontation vs belief modification in persistently deluded patients. Br J Med Psychol 1978; 51: 127-130.

4 Garety PA, Fowler D, Freeman D, Bebbington P, Dunn G, Kuipers E: A randomised controlled trial of cognitive behavioural therapy and family intervention for the prevention of relapse and reduction of symptoms in psychosis. Br J Psych 2008; 192 : 412-423. 
Table 1. Summary of correlation and meta-regression analyses of effect size against 3 demographic and 8 therapist and supervision variables while controlling for CTAM scores. $(\mathrm{N}=24)$

\begin{tabular}{|c|c|c|c|c|c|}
\hline Correlation & $\mathrm{P}$ & $\begin{array}{c}\text { Meta-Regression } \\
\text { coefficient }(95 \% \\
\text { Confidence } \\
\text { intervals) }\end{array}$ & $\mathrm{T}$ & $\mathrm{P}$ & $\begin{array}{c}\text { Partial } \\
\quad \mathrm{r}\end{array}$ \\
\hline
\end{tabular}

Participant Characteristics

Mean Age

Gender (\% male)

$(\mathrm{N}=23)$

$-0.23 \quad 0.30$

$-0.415$

$-0.59$

0.53

$-0.12$

Number of

Hospitalisations

$(\mathrm{N}=14)$

$0.07 \quad 0.81$

$(-1.996,1.166)$

$-0.077$

$-0.96$

0.36

$-0.33$

Having a postgraduate

CBT qualification

$-0.04 \quad 0.84$

$(-0.255,0.100)$

$\begin{array}{lll}-0.96 & 0.36 & -0.33\end{array}$

Therapist Characteristics

Number of years of experience in CBT for psychosis

Number of years of generic clinical experience

Type of core profession (\% clin psych)

$-0.0$

$-0.31$

0.15

$-0.063$

$-0.38$

0.71

$-0.08$

$(-0.411,0.284)$

$-0.31$

0.15

$-.018$

$-0.49$

0.63

$-0.10$

$(-0.093,0.058)$

$-0.015$

$-0.85$

0.40

$-0.18$

$(-0.051,-0.022)$

$0.01 \quad 0.98$

$$
0.032
$$

$0.25 \quad 0.80$

0.05

Quantity of supervision during the trial

$0.45 \quad 0.03$

$(-0.234,0.299)$

0.032

2.03

0.056

0.40

Supervision as group or individual (\% individual)

$-0.31$

0.15

$-0.193$

$-1.61$

$0.12-0.33$

Quantity of training in trial protocol before trial onset

\begin{tabular}{cccccc}
-0.11 & 0.63 & -0.001 & -0.27 & 0.79 & -0.06 \\
& & $(-0.01,0.007)$ & & & \\
0.43 & 0.04 & 0.332 & 2.13 & 0.045 & 0.38 \\
& & $(0.008,0.656)$ & & & \\
\hline
\end{tabular}


